\title{
ESSENTIALS IN THE TREATMENT AND CARE OF THE INVESTING STRUCTURES OF THE TEETH BEYOND THE PREVENTIVE STAGE
}

\author{
By ELBERT J. WEAVER, D.D.S., Milwaukee, Wisconsin
}

(Read before the National Dental Association, Milwaukee, Wisconsin, August 15-19, 1921)

$\mathrm{T}$ ODAY the era of prevention in both medicine and dentistry is at hand. The proportionate number of cases is rapidly increasing, where priventive measures along the line of prophylaxis are working wonderful results in the elimination of the serious phases of dental operations and engineering replacements.

There are numerous forms and clinical manifestations of pyorrhea, and I use this term so generally known just in the way that one would use the word "fever," as a very general term, to bring to our minds one or more of the diseased conditions to which the investing structures of teeth are heir.

Before discussing abnormal conditions of investing structures, we shall be obliged to eliminate the question of nonvital or dead teeth, for we cannot expect to gain headway in the treatment of surrounding structures for the elimination of formative or productive areas of lowgrade infection in the mouth, without first satisfying ourselves, as far as possible, that there are no foci of infection remaining at any of the non-vital root ends.

The extraction of devitalized teeth at the present time in view of rational ideas now held by the majority of men in the profession finally comes down to a question of common sense and good judgment.
The removal of infected devitalized teeth is not the first step in the march of treatment for the eradication of infection present in the structures surrounding the teeth, but the consideration of that procedure at this time gives us a premise to build on, which will be taken up again a little later, and brings us to the first step in the treatment of a case of advanced periodontoclasia or pyorrhea which presents.

The first essential is to eliminate from the system the field for production of lowgrade infection in and about the processes of the maxilla and mandible. In attempting to do this we must have in mind the ophthalmic index or the state of health of the patient.

1. Relation of the Physical Condition to the Local Manifestations.-The first essential in the march of treatment is the consideration of the physical condition of one presenting with an advanced case, which usually means extensive bone destruction. The departure from normal in the appearance of the gum tissues and the destruction of bone has of course been made manifest through careful radiography and clinical examinations combined with a correct history of the case, two important aids in the consideration of this essential.

General treatments and diets will vary, 
as for instance in the diabetic variety; where traumatism is the cause mechanical interference must remove the abnormal stress; but prophylaxis or surgical treatment as indicated is the foremost factor locally in successfully handling all varieties.

We cannot lay too much stress on the importance of a perfect set of films in these cases. He must study them carefully, and re-check them at different angles, the different density in different kinds of bone structure, various bony formations that throw shadows, and the foramina and canals that sometimes at a casual glance appear to be rarefied areas.

The periodontist should record a history in these cases as we deem it important for future reference and for our own records, but it does not need to be too technical, especially as we expect a more detailed report from the medical diagnostician. However, we should know and record the age, history of previous diseases and recent disabilities and symptoms, occupation, which is important as it has to do with the etiology of the disease and gives an idea of the conditions under which the patient is living, in regard to exposure and amounts of exercises taken; also what parts of the body are being exercised, whether occupation is principally physical or mental and whether food can be taken regularly, etc. Something on this plan, or a more elaborate history if desired, should be recorded.

2. Nonvital Teeth.-The patient then calls upon the diagnostician for a complete physical and systemic examination. With this report in hand we are ready to turn back to the question of nonvital teeth.

We believe even in a case showing normal or nearly normal findings that teeth with rarefied areas present as well as teeth with very little bone attachment which are loose, should come out. In short we should not attempt to save a tooth which is a menace to the patient's health. We should not consider root resection in these advanced cases, even of normal findings.

The non-vital teeth showing areas and the hopelessly loose one, having been dispensed with, the patient will wish to know what the prognosis is in his particular case. There is an occasional exception, but our answer to that question can safely depend on what in our judgment we can hope to accomplish locally with surgical or other treatments and upon the vitality of the part, which in a measure is the reflection of the whole general systemic condition. In this connection our records show cases of interest concerning the value of systemic examinations. The following are a few cases:

Case A: Young woman nineteen years old, presenting with results of X-ray and clinical findings, showing deep pyorrhea sinuses on mesio-buccal surfaces of upper molars, also on disto-lingual of another lower molar. There were no deposits of any kind, simply an infectious destruction of periosteum, peridental membrane and bone. The history was difficult to get, and was finally obtained from the mother. The physical condition was a large factor in this case, if not the whole reason for this destruction of bone, and was probably of the anemic type. Physical findings revealed conditions of myasthenia and neurosthenia, leucocytosis, secondary anemia with autointoxication, in fact, the girl had nearly lost the sight of one eye. After intensive medical treatment, during which period the mouth condition had been taken care of, the patient gained in weight back to nearly normal and unless there is a recurrence of the physical symptoms, there is little chance of the destruction of bone beyond the normal.

Case B: Woman of forty-five under 
observation and local treatment about nine years, two teeth had been previously removed and local surgical treatment carried out. We were aware in this case, of gastrointestinal toxemia. Upon each return call for prophylactic care, about every three or four months, case always revealed gingival inflammations, sometimes infectious and large amounts of calcareous deposits. This case was despaired of and an attempt was made a great number of times to get the systemic findings, but without success. Finally, an acute gall-bladder attack necessitated an operation. Patient was not seen until nearly six months after same and the change in the condition of the gums was marvelous. Simple prophylactic treatments every four months are now keeping the mouth in healthy, normal condition; in fact, it would hardly be believable that it was the same case.

Case C: Woman of forty-five presented case in which X-rays showed extensive destruction of bone. Clinical examination revealed suppurative condition of gum tissue, periodontal membrane, periosteum and bone. Deep bluish color marked the location of sinuses. Local loss of tone very marked. Operation few years previous, and case was delayed for physical examination; urine, very fair; blood pressure, all right; heart and lungs, negative; no hemorrhoids; neurotic and history showed nervous exhaustion; tonsils had been removed, slight catarrhal condition pharynx and naries; postpharyngeal membrane lacked muscle tone; uterus, convex, almost obliterated from retraction; history of an ovariectomy complete on one side. Patient showed number of other technical complications, but this was the seat of a large amount of low-grade infection and if not the cause of the mouth condition, it had a strong effect on the vitality of the part.

Case D: Woman of forty-eight, one of the cases that taught us the advisability of clinical examinations, apparently was of the auto-intoxication and constipation type systemically. Mouth was thoroughly treated surgically and prophylactically, but we were absolutely unable to get the gums in any condition of health which would last for more than two weeks. Inflammations would always reoccur accompanied sometimes with infections, and fungous growth interproximally on the gingival margins. At the time this case was in our hands we did not have a very good history as an attempt to obtain a physical examination failed. Suddenly the woman had a stroke of paralysis and was removed to a sanitarium. We have no record of the systemic abnormality, but wish to stress the futility of local treatments alone in a case of this nature.

Case E: Young woman of twentyfour, mild case of gingivitis with acute inflammation. Gave history of failure to respond to vapor treatments, constipation and mild attacks of indigestion. Prophylaxis failed to bring desired results until physical examination revealed the necessity of tonic and diets and increased water intake. Within a short time the local inflammations began to subside and the mouth will retain its good condition unless the patient is careless and allows the general symptoms to return, at which times the recurrence of the local condition is noticeable; but it is less noticeable each time as the vitality of the investing structures. is improving from both local and systemic measures.

Case F: Woman of fifty has been in our care about ten years; there is great difficulty to maintain gum tissue in state of health and keep teeth clear of deposits. Patient simply refused to have physical examination but was willing to call every month for prophylactic work. Finally suffered an attack of hemorrhoids 
and had same surgically treated. Within a month's time improvement in condition of mouth was very marked. We feel sure that had we been insistent enough about getting physical findings in this case some years ago, we would have saved a large amount of trouble and have been spared much of worry.

In citing these cases, I do not wish to be understood as assuming that all the local abnormalities in these cases were the result of the general systemic condition, but from our clinical experience it must be concluded that it is wise to have more in mind than just the local manifestations in treating these cases.

3. Pre-treatment.-We will take as an example a case where the color of the gum is an abnormal dark red or verging on bluish in the locations of deep sinuses, with or without marginal inflammations. Radiographs show considerable amount of bone destruction and either serumy pus or bacteria charged serum, or the heavier varieties of pus, with visible deposits or apparently little. Whatever the variety or form of manifestation or whatever nomenclature one may care to use in describing it, the local treatment is similar except perhaps in its intensity.

Here one may make use of laboratory tests to ascertain which forms of bacteria are present locally. In the large number of bacteria present at times in the mouth flora, we have probably to deal more especially in these cases with the different forms of staphylococcus and streptococcus, unless we are dealing with Vincent's disease, which we will not consider at this time. The internist may deem an autogenous vaccine advisable in a case showing streptococcus infection in the blood stream and one should know how to prepare and preserve a smear or specimen, a fact which emphasizes the cooperation between the diagnostician and the dentist. The pre-treatment varies only in its duration for different cases as follows:

The mouth is sprayed with a strong antiseptic and the visible calcareous deposits removed with a cursory scaling. 'The teeth are then cleaned with an engine brush or scrubbed with a toothbrush in the hands of the operator. We then subject the mouth to a massage suction treatment, using suction cups for the purpose of draining and cleansing the pockets and in a measure emptying the gorged blood vessels. This is done twice or three times a week for from two to three weeks. Our object in this treatment is to produce hyperemia and to revitalize the parts. The toothbrush in the hands of the operator, carefully scrubbing and rotating the brush against the gums at each visit of the patient, appears to give good results, although the suction produces deeper stimulation.

Before starting our surgical work at this time, it is well to scan the occlusion. By laying the finger along the buccal or labial surfaces of the teeth, any tooth that strikes hard enough to produce serious trauma can be detected by moving the jaws laterally with the teeth in occlusion. These should be ground, and the continuity of the grinding and cutting surfaces kept in mind with the idea of completing this operation when the surgical work has been finished.

After each suction treatment, a medicament is used.

4. Instrumentation.-We are dealing with advanced cases and we shall consider the use of instruments in that light more than from the standpoint of simple prophylactic work.

A small locality of from two to four teeth having been selected as our field of operation, we start with one of a set of four "push and pull" scalers. The other three are bent to about the angle of the greater curve and these four will reach 
any surface of any tooth in the mouth comfortably. These instruments are for the rough work and are also very useful for subsequent prophylactic work. Next we go between the approximal surfaces with a scaler formed like a scimitar, that will often catch particles missed with the others. Then the planes with which all the surfaces are reached down to the bottom of the pockets, thoroughly but carefully; it should be remembered that they are planes and should be used in general parallel to the long axis of the tooth. It is best to prove up the work with a pair of files, which cover every surface of practically every tooth in the mouth. They will detect any little particle of deposit or roughness left and are also used in the bottom of the sinuses and along the rough edges of the process as curettes, smoothing the bone and dislodging the germ nests. With this operation we also dislodge the lateral cysts which are so often found.

This method has appeared to bring results unobtainable from just planing and polishing the tooth surfaces. The instrumentation is finished by carefully going over all surfaces under the gum margin and a short distance above with files, whose surfaces are not too rough; they should be used more as burnishers. Files which have been used seem to answer the purpose admirably. Gum resection is sometimes necessary, especially between the posterior teeth. Experience has taught that we can save more gum substance by constant stimulation which builds up attachment and bone structure from below. Where the fibrous gum tissue is too loose or thick to be overcome by this treatment, it can be burned down a number of times with phenol sulphonic acid, applied with cotton wound on a treatment point, until the part is hard and firm.

Following this comes the polishing which can be done with disks, strips, tape, moose hide wheels, points of wood and other materials used with fine abrasive, but one should be cautioned against too free use of stones. The least cutting of the tooth surface possible which leaves a smooth surface tends to minimize the bugbear of sensitiveness.

5. Occlusion.-We return again to occlusion and contact points. The teeth must rock in occlusion without any undue stress on any one or a few teeth. Enough time spent with wax articulating paper, and stones with the thought in mind of the continuity of the tooth grinding and cutting surfaces, will produce good results. The important thing is to watch carefully as the years pass, and detect before any damage is done, any tooth that may again be striking too hard. Our rule is always to try the occlusion in pyorrhea mouths returning for subsequent prophylactic work. The value of proper contact points is well understood and only needs to be mentioned in passing.

6. Post-Treatment, or After-Care by the Operator-The patient is required to call once or twice a week for at least a month, sometimes for a much longer time, for stimulation treatments, which are given by the dental assistant and consist of suction treatments, followed by gently wood-sticking against the gums or slightly under them. This treatment was discovered to be of value, accidentally, in treating sensitive teeth, and for such teeth we know of no better treatment than wood-sticking with zinc iodide, or, in severe cases, coating with solution of tincture of benzoin and tannic acid, mixed into a thick paste and applied after drying the tooth surface and allowed to stand free from moisture for at least five minutes.

Formula for zinc iodide solution: zinc iodide, 15 parts; water, 10 parts; iodin, 25 parts; glycerine, 50 parts. 
In treating some very sensitive teeth around which there had been deep pockets by rubbing every few days with zinc iodide, it was very noticeable that there was a rapid improvement in the structure and appearance of the gums, so much so that the treatment was tried in cases where there was no sensitiveness, with good results, and is now included in our regular treatment.

7. Care by the Patient at Home.-As the patient returns for these stimulating treatments, his education as to the home care is undertaken. First the brushing is described and illustrated. Methods of forcing the bristles between the teeth with a slight rotary motion can be recommended, especially where there are good approximal spaces. The sweeping motion brushing the whole surface of gum tissue and tooth surface toward cutting edge and the circular method in different hands all seem to produce the desired results. Massage produced by placing the tip of the forefinger interproximally at the gum margin and allowing the finger at point of contact to move slightly, rotating the hand through a much larger arc, all around the mouth, inside and out, is a fine massage for bone and tissue building. We insist, though, upon our patients finishing the cleaning operations with the use of wide dental floss charged with a slight abrasive, and we attempt to teach just as definite technic in the use of the floss as we use in the instrumentation. The bottle containing floss is held in the right hand, 10 or 12 inches of the floss being drawn out. The loose end is held in the left hand and drawn around the third and fourth fingers of same hand. Then it is drawn across the end of the thumb and index finger or around the index finger and over the end of second finger, depending upon what particular place in the mouth it is to be used. With the right hand containing the bottle, the floss is wound around index finger until only about $1 / 2$ or $3 / 4$ of an inch separates the finger of the right hand from finger or thumb of left hand. The floss is changed from index finger of right hand to thumb depending also on where it is to be used. We are ready for application in the mouth.

We start on left upper and keep fingers or thumbs inside cheeks, back of last tooth, then between molars, etc., cleaning and polishing the distal of the mesial tooth and the mesial of the distal, then moving the floss mesially and distally across the interproximal gum tissues. There can be no possible harm done if the contact points are left so that the floss will go through snugly but easily. If the fingers are kept about the distance apart that the teeth are wide it is almost impossible to strike down on gums. Under bridges a short curved bodkin thru the eye of which the floss is threaded, is used.

This method has given gratifying results. The patient is taught the correct technic and then given floss to take home. At the next visit any errors that might have occurred in its use at home are corrected. We advise the patient to stand in front of a mirror in order to see what he is doing in brushing and in the use of the floss.

Finally one of the most vital points in the permanency of the results which we have obtained in these cases of advanced pyorrhea conditions is the regularity with which the patients return for prophylaxis. With proper care on our part and with conscientious attention given at home, there is a steady improvement.

\section{SuMMary}

1. The Relation of the Physical Condition to the Local Manifestations can be summed up in closer relation between the medical diagnostician and the den- 
tist, as it is in conjunction with him that we are attempting to eliminate fields for the production of low-grade infection. The mouth is not the only field for production and the local condition may be seriously influenced by other physical ailments.

2. Any nonvital teeth that are a menace to the patient's health should be sacrificed. Some sinuses of an apparent pyorrhea nature have their origin in infected root ends.

3. Pre-treatment. The tissues should be put into a revitalized state before instrumentation is attempted.

4. Instrumentation includes a definite technic and mode of procedure; gum resection should be resorted to just as little as possible.

5. Occlusion. A very important essential and one, which if neglected, will doom to failure the most care-taking application of the other essentials.

6. Post-treatment or After Care by the Operator. Cases should not be pronounced finished until the operator is sure that the surrounding tissues show no inflammation and are hard and firm.

7. Care by the Patient at Home. No matter how well the periodontist has performed his work the case should not only stay in the condition in which it was discharged, but it should improve as the years pass, providing this important essential is painstakingly performed by the patient.

\section{Discussion}

Justin D. Towner, Memphis, Tennessee: I wish to emphasize first of all the importance and the necessity of an examination, both local and physical. We must remember that no examination is complete without inquiring into the case history of the patient, for that is the only means we have of getting any idea of a difficult pathology. It is not necessary to go into the details of the local or physical examination, but that very few dentists are competent, either professionally or legally, to make a physical examination or to render proper judgment in that particular. Therefore, we must depend upon our medical brother and all such cases should be referred to the internist or proper diagnostician.

Diagnosis comes next, for an accurate diagnosis is essential for careful and efficient treatment.

The next requisite is the pre-treatment. That is very essential. I differ with the essayist's opinion in that $I$ begin on the occlusion. Granting that the case belongs to the periodontists, I begin on the occlusal surface and the first thing is to remove traumatic occlusion, or any defect of occlusion that is influencing the disease.

The next step is to thoroughly treat and cleanse the crowns of the teeth, for that is presurgical. There are certain requisites in all cases and certainly thorough cleansing is necessary to remove infection before any proper surgical interference can take place. I must impress upon you the necessity of standardization of your procedure, whether it is your own or that of someone else. The cutting edge of the files and scalers comes off at a right angle, because in some instruments there is almost a millimeter between the cutting edge and the end of the instrument and you cannot get to the bottom of your pocket. You have to go under if you are going to remove the disease.

Dr. Weaver has a fifth prerequisite, occlusion. I advocate it as the first step in the treatment of local disease because it has that much power and assumes that much importance in the treatment of trouble. The post-treatment is very essential.

The trouble with periodontists today is this: they assume too much responsibility for cure. Three factors enter into this: one is the physical resistance or vitality of the patient. First the vital resistance, next the personal care taken of the mouth and, third, the periodontal treatment. If that is true, we cannot rightly assume more than one-third of the responsibility for the care of any case we treat.

The essayist's conclusion is very well taken. All work is only incidental to health and if you chose to say that it is incidental to the health of local conditions, then it must have a bearing on the physical condition because the oral cavity has such a large bearing on the physical make-up, whether in health or disease.

A. F. Isham, Buffalo, New York: I have been using this method of cleaning teeth in my own mouth for ten years. Previous to that time I saw a prescription in one of the magazines, of which the only gritty constituent is chalk. My method is to use this tooth powder and then before I rinse my mouth I take the tape and go between the teeth. Something has occurred in my mouth. I can take a quill toothpick and go between all the teeth. I do not know whether this is only the normal con- 
dition due to advanciig age or whether I have worn them off fro:s the cleansing. I wonder if there are any other cases similar to mine.

H. J. Leonard, Minneapolis, Minnesota: I was particularly delighted with the case mentioned in which the factors had been traced back to those that had caused the lowered resistance on the part of the patient. They cannot always be traced.

The simplest method that we can use to raise the resistance of the tissues and to reduce the irritation is the one we should select. We want to use the least amount of time in treating our patients. We want to treat the largest number of patients and any method that involves a greater number of steps than is absolutely necessary should be abandoned. I take exception to the methods of pre-treatment, methods which have to have a considerable amount of time spent on them, because I feel they are not necessary to get the results we should expect. In teaching periodontia to students where we want a large number of men to do the work after they graduate, at least in its simpler aspects, we have to eliminate all unnecessary steps. I feel that we as periodontists should make great effort to discourage the use of medicaments which take unnecessary time to apply and whose value seems at best doubtful.

Dr. Weaver (closing): In these advanced cases I consider it absolutely necessary that the patient has a thorough physical examination before the local operations are attempted.

We are also finding that there are two classes of physicians, those who are treating people who are very sick and confined to their bed, and the class who are doing preventive work, by attending people who are up. It is the latter class that we are attempting in the main, to deal with. The diagnostician also is beginning to thank the periodontists for the strong influence on patients in their hands toward preventive work along medical lines.

In regard to low-grade infection there are a number of localities for the formation of these infections, as the teeth, the cranial sinuses, the digestive tract, the prostate gland, genitourinary organs, etc. We must remember that the teeth are not the only source of low-grade in.ection.

In regard to occlusion: the first thing done is to scan the occlusion and do the rough work, to the extent that there are not one, two or more teeth which are pounding entirely too hard.

I want to impress the importance of curetting the diseased soft edges of the surrounding alveolar process and soft tissues, as it is difficult to believe what will be dug out at times, in the way of diseased bone, lateral cysts, etc.

In regard to Dr. Isham's question about the dental floss: I have been using the floss in the way I have described for about five years and I have noticed in a good many instances and in my own mouth that what he speaks of happens. We all are growing old and owing to the lowered vitality in other parts of the body as well as in the mouth, the bony structure around the teeth begins to deteriorate. As these structures are transitory structures and are end organs, naturally they sometimes deteriorate quite early in life. Using the dental floss regularly seems to harden the tissue and keep it in closer conjunction with the bone and as the bone is absorbed, this massage causes the tissues to follow it down, making more interproximal spaces between the teeth.

In regard to Dr. Leonard's remark concerning too much pre-treatment: it must be remembered that I am discussing serious advanced periodontal cases and personally, I consider this work very important in these serious cases of long standing, because the local resistance is very much lowered and the healing process takes place so much more rapidly when these tissues have been in a measure, revitalized before surgical work is commenced. 